\title{
Application of Astatic State Observers in Electromechanotronic Modules
}

\author{
Nikolay Gnezdov, Ivanovo State Polytechnic University, Aleksey Kolganov, Ivanovo State Polytechnic University, \\ Sergey Lebedev, Ivanovo State Polytechnic University
}

\begin{abstract}
The authors offer a synthesis method of state observers with high-order astatism. There are no errors caused by the action of arbitrary external disturbances in reduced variables of such observers. The observers also restore the instantaneous values of disturbances. The authors present a calculation method of ultimate realizable dynamics of observers during their realization in microprocessor control system which calculates in numbers with fixed point without overflowing of variables. The description of experimental installation and software is given.
\end{abstract}

Keywords: digital signal processors, drives, observers, state estimation

\section{INTRODUCTION}

The task of ensuring "informational transparency" of the controlling object is one of the problems while creating energy-efficient electromechanotronic modules and systems. Its solution can be obtained either using multiple sensors or using computing devices for calculating the indirect variables, which include state observers [1]-[3].

Constructive, technical and economic restrictions, impossibility of measurement of some important variables by means of sensors, entering inadmissible static and dynamic errors by sensors are obstacles of sensor utilization.

State observers allow to get information about the object state and disturbances according to affordable input and output information, as well as the parameters of its mathematical model [4]-[6].

Development tendencies of electromechanotronic systems, relating to the observers, are: expended using of observers in the construction of control systems of various objects, improving the quality requirements for the estimating of the variables (accuracy, speed, range), increasing the systems noise immunity, detailing the object mathematical description [7].

Intensive using of the observers and the incorporation of new complexity factors while describing the control objects and modes of their work attach the researchers' interest to methods, techniques, synthesis algorithms and observers setting up, improving the quality of objects' coordinates estimation. The significant impulse to expand the using of the observers in electromechanotronic systems, particularly in high-speed AC drive systems, made the transition of control devices on the digital, microprocessor element basis [8].

At present, productivity and performance of DSPmicrocontrollers for motor control allow to realize the digital implementation of state observers, synthesized in the continuous systems.

\section{Synthesis Methodology of THE OBSERVERS}

The presence of external disturbances in the object makes a forced component in the transition process of estimation a variable by the state observer. When disturbance is constant, the static error of recovering the variable takes place, that is increased linearly with the growing disturbances. With a capital fluctuation the static torque error varies according to the harmonic. Errors in the feedback channel as an object can lead to unacceptable errors in the management of state facilities.

The authors suggest a method of assessing the observability and the synthesis of astatic observers with the action on the object of unlimited number of both permanent and variable disturbances [9], [10]. The methodology is based on formalization of information pertaining to the disturbance in the form of a mathematical model and expansion of the model of object management through the model disturbance. Depending on the objectives assigned to the control system, the disturbance can be represented with varying degrees of detail.

For example, the model of quasi-stationary scalar disturbance is $d z(t) / d t=0$, the model of scalar disturbance with quasi-stationary speed of change is $d \dot{z}(t) / d t=0$ or $d^{2} z(t) / d t^{2}=0$, and etc.

In general, when the vector (multivariate) nature of each types of disturbances influencing on the object is added the object model of well-known state equations [5] as well as state equation of disturbance. As a result, the state equation and equation of output will be presented as block matrices of parameters and an extended state vector, which include the vectors of each type of disturbance:

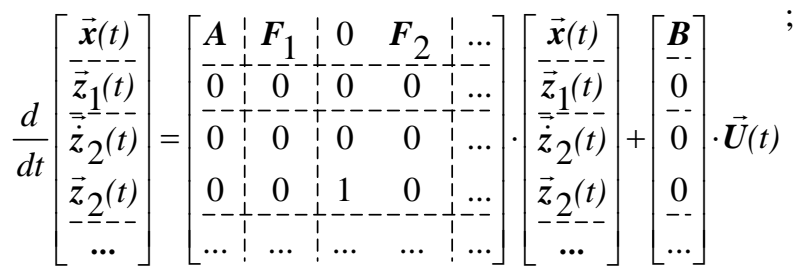

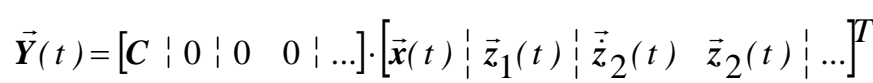

where $\vec{z}_{1}(t)$ and $\boldsymbol{F}_{1}$ are the vectors of quasi-stationary disturbances and the matrix of its dimension parameters $n \times k$; 
$\vec{z}_{2}(t)$ and $\boldsymbol{F}_{2}$ are the vector disturbances with quasispeed changes and the matrix of its dimension parameters $n \times l$.

For example,

$\boldsymbol{A}_{z}=\left[\begin{array}{c:c:cc:c}\boldsymbol{A} & \boldsymbol{F}_{1} & 0 & \boldsymbol{F}_{2} & \ldots \\ \hdashline 0 & 0 & 0 & 0 & \cdots \\ \hdashline 0 & 0 & 0 & 0 & \cdots \\ 0 & 0 & 1 & 0 & \cdots \\ \hdashline \cdots & \cdots & \cdots & \cdots & \cdots\end{array}\right], \quad \boldsymbol{B}_{z}=\left[\begin{array}{c}\boldsymbol{B} \\ \hdashline 0 \\ \hdashline 0 \\ 0 \\ \hdashline- \\ \cdots\end{array}\right]$,

$\boldsymbol{C}_{z}=\left[\begin{array}{lllllllll}\boldsymbol{C} & 0 & 0 & 0 & 0 & 0 & \ldots\end{array}\right]$

Then the Kalman observability matrix [5] is for the system (1) with using (2):

$$
\begin{array}{r}
\boldsymbol{V}_{z}=\left[\begin{array}{l:l:l:l}
\boldsymbol{C}_{z}^{T} & \boldsymbol{A}_{z}^{T} \boldsymbol{C}_{z}^{T} & \left(\boldsymbol{A}_{z}^{T}\right)^{2} \boldsymbol{C}_{z}^{T} & \ldots \\
& \ldots & \left(\boldsymbol{A}_{z}^{T}\right)^{(n+k+l)-1} \boldsymbol{C}_{z}^{T}
\end{array}\right]
\end{array}
$$

Observability condition is the equality of the observability matrix rank and the sum of the objects orders and the dimensions of the disturbance vectors

$$
\operatorname{rank} \boldsymbol{V}_{z}=n+k+l+\ldots
$$

It allows to estimate the recovery opportunities for object state vector and all the disturbance vectors.

The synthesis of astatic state observers with the disturbance model can be made by the method of standard coefficients [5]. The equation of state and the characteristic equation of astatic observer have the form:

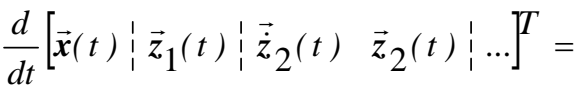

$$
\begin{aligned}
& =\boldsymbol{A}_{z} \cdot\left[\begin{array}{l:l:l:l:l}
\overrightarrow{\boldsymbol{x}}(t) & \vec{z}_{1}(t) & \vec{z}_{2}(t) & \vec{z}_{2}(t) & \ldots
\end{array}\right]^{T}+\boldsymbol{B}_{z} \cdot \overrightarrow{\boldsymbol{U}}(t)+
\end{aligned}
$$

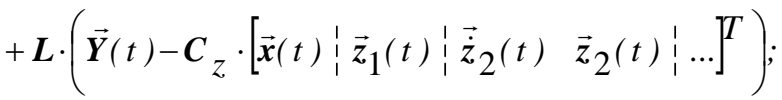

$$
\begin{aligned}
& \operatorname{det}\left(s \cdot \boldsymbol{I}-\boldsymbol{A}_{z}+\boldsymbol{L} \cdot \boldsymbol{C}_{z}\right)=0 \text {. }
\end{aligned}
$$

Here, as in the control devices we can apply the concept of astatism degree. Then the observer with the model of quasistationary disturbances is called as a state observer with the first order astatism (SOA1), the observer with the disturbance model of quasi-stationary speed of change is a state observer with the second order astatism (SOA2) and etc.

The state observer with the second-order astatism was synthesized for viscoelastic two-mass electromechanical system using the method described above. The block diagram of the observer is shown in Fig. 1.

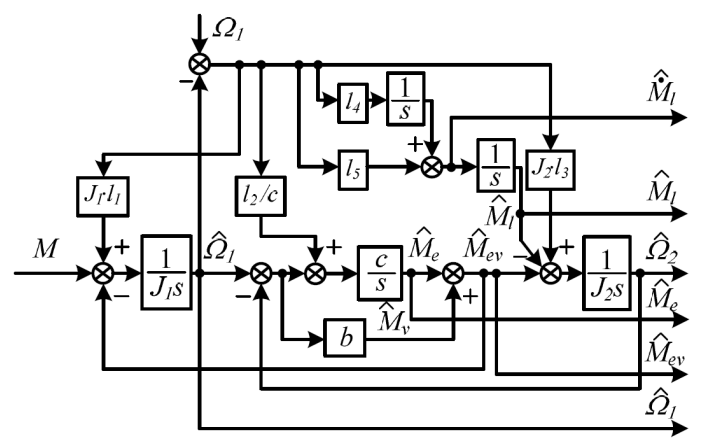

Fig. 1. The block diagram of the observer with the second-order astatism.

The use of state observers with a high (second, third, etc.) order astatism theoretically gives no errors in the residue of an observer while appropriate type of load acting on the object (ramp, parabolic changing, etc.). Furthermore, the increasing of the observer order astatism can reduce the ratio of its speed to the regulator speed, and it allows to increase the system bandwidth.

In addition, the proposed observers provide an assessment of the instantaneous value of mechanical load on the electric drive shaft when it is changed by an arbitrary law or with a specific frequency band, which gives the way for applying the combined control principles with using the feedback on the disturbance.

\section{OBSERVERS IMPLEMENTATION IN MICROPROCESSORS}

To implement the observer in the digital control system of electric drives, we have to calculate the equations by one of the numerical integration methods. For example, for the Euler method differential equation of the observer with the 2-nd order astatism (Fig. 1) in standard coordinates notations of the electric drive and the observer coefficients, have the form (7).

In (7) initial values are given: $\tilde{\Omega}_{1}(0), \tilde{M}_{e}(0), \tilde{\Omega}_{2}(0)$, $\tilde{M}_{e v}(0), \tilde{d M}_{l}(0), \tilde{M}_{l}(0)$.

The calculation period $\tau$ is determined by calculating the observer permissible error of calculations.

To calculate (7) in the microprocessor it must be determined:

- dimension of variables;

- scaling factors used for operations with variables of different dimensions;

- coefficients in (7), used in the calculation in the microprocessor.

The dimension of the variables determines the characteristics of digital implementation:

- accuracy of variables reconstruction;

- bandwidth of the observer, in which an overflow of microprocessor registers, which means the variables of the observer, does not occur.

On the other hand, to realize the bandwidth of the observer we can determine the required dimension of the variables. So, there are direct and inverse approach to the definition of the variables dimension and the calculation of the coefficients in differential equations. 


$$
\begin{aligned}
& \tilde{\Omega}_{1}(k+1)=\tilde{\Omega} 1(k)+\tau \cdot\left(\frac{1}{J_{1}}(M(k)-\right. \\
& \left.\left.-\tilde{M}_{e v}(k)\right)+l_{1}\left(\Omega_{1}(k)-\tilde{\Omega} 1(k)\right)\right) \text {; } \\
& \tilde{M}_{e}(k+1)=\tilde{M}_{e}(k)+\tau \cdot\left(c \cdot \left(\tilde{\Omega}_{1}(k)-\right.\right. \\
& \left.\left.-\tilde{\Omega}_{2}(k)\right)+l_{2}\left(\Omega_{1}(k)-\tilde{\Omega_{1}}(k)\right)\right) ; \\
& \tilde{\Omega}_{2}(k+1)=\tilde{\Omega}_{2}(k)+\tau \cdot\left(\frac { 1 } { J _ { 2 } } \left(\tilde{M}_{e v}(k)-\right.\right. \\
& \left.-\tilde{M} l(k))+l_{3}\left(\Omega_{1}(k)-\tilde{\Omega_{1}}(k)\right)\right) \text {; } \\
& \tilde{M}_{e v}(k+1)=\tilde{M}_{e}(k+1)+ \\
& +b \cdot\left(\tilde{\Omega}_{1}(k+1)-\tilde{\Omega}_{2}(k+1)\right) ; \\
& \tilde{d M} l(k+1)=\tilde{d M} l(k)+ \\
& +\tau \cdot l_{4} \cdot\left(\Omega_{1}(k)-\tilde{\Omega} 1(k)\right) ; \\
& \tilde{M}_{l}(k+1)=\tilde{M}_{l}(k)+\tilde{d M} l(k+1)+ \\
& +\tau \cdot l_{5} \cdot\left(\Omega_{1}(k)-\tilde{\Omega} 1(k)\right),
\end{aligned}
$$

where $\tau$-observer calculation period;

$k, k+1-$ numbers of calculation step.

To implement the observer in the digital control system it is necessary:

- to calculate the coefficients in (7);

- to identify bandwidth above which the restriction of the variables are.

We obtain system (8) using the microprocessor dimensions for variables in equations (7).

To determine the maximum possible bandwidth of the observer $f_{b w \text { max }}$ at a given period of calculation and the dimensions of the variables the authors have developed a program in one of the systems of computer mathematics.

The program takes into account the ratio of the root mean square $\omega_{0 s}$ and bandwidth $f_{b w}$ for a binomial distribution of the roots of the observer characteristic equation [11].

When the objects parameters are $J_{1}=0,055 \mathrm{kgm}^{2}$, $J_{2}=0,277 \mathrm{kgm}^{2}$,

$$
c=553,633 \mathrm{Nm} / \mathrm{rad}
$$

and

$b=0,83 \mathrm{Nms} / \mathrm{rad}$, the calculation period is $\tau=1 \cdot 10^{-3} \mathrm{~s}$ and dimensions of the variables is $\Omega: 0,000488 \mathrm{rad} / \mathrm{s}$ (4-byte sign variable), $M: 1,192 \cdot 10^{-7} \mathrm{Nm}$ (4-byte sign variable), the following values are: $f_{b w \max }^{S O A 2}=9,7 \mathrm{~Hz} \quad$ and $\omega_{0 s \text { max }}^{S O A 2}=188 s^{-1}$.

$$
\begin{aligned}
& \left\{\begin{aligned}
\tilde{\Omega}_{1}^{*}(k+1)= & \tilde{\Omega}_{1}^{*}(k)+k_{m 1}\left(M^{*}(k)-\tilde{M}^{*} e v(k)\right)+ \\
& +k_{\tau 1}\left(\Omega_{1}^{*}(k)-\tilde{\Omega}_{1}^{*}(k)\right) ;
\end{aligned}\right. \\
& \tilde{M}_{e}^{*}(k+1)=\tilde{M}_{e}^{*}(k)+k_{\Omega 1}\left(\tilde{\Omega}_{1}^{*}(k)-\tilde{\Omega}_{2}^{*}(k)\right)+ \\
& +k_{\Omega 2}\left(\Omega_{1}^{*}(k)-\tilde{\Omega}_{1}^{*}(k)\right) \text {; } \\
& \tilde{\Omega}_{2}^{*}(k+1)=\tilde{\Omega}_{2}^{*}(k)+k_{m 2}\left(\tilde{M}_{e v}(k)-\tilde{M}_{l}(k)\right)+ \\
& +k_{\tau 2}\left(\Omega_{1}^{*}(k)-\tilde{\Omega}_{1}^{*}(k)\right) ; \\
& \tilde{M}_{e v}^{*}(k+1)=\tilde{M}_{e}^{*}(k+1)+ \\
& +k_{\Omega 3}\left(\tilde{\Omega}_{1}^{*}(k+1)-\tilde{\Omega}_{2}^{*}(k+1)\right) ; \\
& \tilde{d M}^{*} l(k+1)=\tilde{d M}^{*}(k)+k_{\Omega 4}\left(\Omega_{1}^{*}(k)-\tilde{\Omega}_{1}^{*}(k)\right) \text {; } \\
& \tilde{M}^{*} l(k+1)=\tilde{M}_{l}^{*}(k)+\tilde{d M}_{l}^{*}(k+1)+ \\
& +k_{\Omega 5}\left(\Omega_{1}^{*}(k)-\tilde{\Omega}_{1}^{*}(k)\right),
\end{aligned}
$$

where $k_{m 1}=\frac{m_{\Omega}}{m_{M}} \cdot \frac{\tau}{J_{1}} ; \quad k_{m 2}=\frac{m_{\Omega}}{m_{M}} \cdot \frac{\tau}{J_{2}} ;$

$$
\begin{aligned}
& k_{\Omega 1}=\frac{m_{M}}{m_{\Omega}} \cdot \tau \cdot c ; \quad k_{\Omega 2}=\frac{m_{M}}{m_{\Omega}} \tau l_{2} ; \quad k_{\Omega 3}=\frac{m_{M}}{m_{\Omega}} b ; \\
& k_{\Omega 4}=\frac{m_{M}}{m_{\Omega}} \tau l_{4} ; \quad k_{\Omega 5}=\frac{m_{M}}{m_{\Omega}} \tau l_{5} ; \\
& k_{\tau 1}=\tau l_{1} ; \quad k_{\tau 2}=\tau l_{3} ; \\
& m_{M} \text { - torque dimension; } m_{\Omega}-\text { speed dimension. }
\end{aligned}
$$

Similar calculations performed for full state observers and observes of the first order astatism [9], have given values for: $f_{b w \max }^{S O F}=329,2 \mathrm{~Hz}, \quad \omega_{0 s \max }^{S O F}=4064 \mathrm{~s}^{-1} \quad$ and $f_{b w \max }^{S O A 1}=46,2 \mathrm{~Hz}, \omega_{0 s \max }^{S O A 1}=701 \mathrm{~s}^{-1}$.

As we see, it has to reduce the bandwidth at times while increasing the order of the observer to comply the conditions of its work in linear zone. At this time it cannot fulfill the specifications requirements for the dynamics and accuracy of recovery variables. The solution is to increase the dimension of the variables, or reduce the period of calculation. Both ways are connected with the higher cost of the hardware control system. In this way, it is rational to use not only more powerful microcontroller, but the transition to a floating-point computation. Recently, the products, including floating point unit, have appeared on the market of DSP-microcontrollers. Texas Instruments (TI) is the leader company in this way. A series of TMS320F2833x microcontrollers has a capacity of up to 300 million floating point operations per second. 
Therefore, DSP-microcontroller TMS320F28335 was chosen for the creation of the experimental setup (Fig. 2, 3).

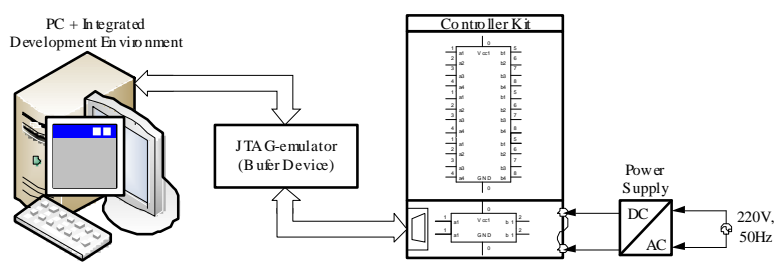

Fig. 2. The functional diagram of the experimental setup.

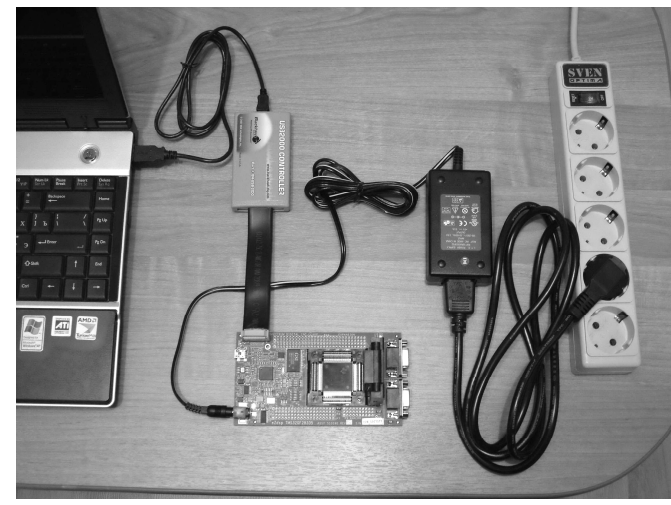

Fig. 3. The experimental setup.

\section{IV.EXPERIMENTS}

The aim of experimental research was to test performance and properties of the observers, as well as the assessment of their applicability in the real electromechanotronic modules. The main element of the experimental setup is a debug card, which on the one hand is connected to DC power supply, and on the other hand is connected to a personal computer through an intermediate device (JTAG-emulator or converter "USBCAN"). Development of software for the experiments was carried out in two stages. First, using a Embedded Target for TI C2000 DSP tool simulation model of "object - state observer" has been translated to code in $\mathrm{C}$ language, and then adjustment of received code was carried out according to the debug board and TMS320F28335. Researches made in this field have confirmed the efficiency and quality of the observers, obtained by the proposed techniques.

\section{CONCLUSIONS}

The use of described astatic state observers provides no errors in the estimated state values including load torque while appropriate type of load acting on the object (ramp, parabolic changing, etc.). Such observers can be applied in electric drives with significant mechanical load on the shaft when it is changed by an arbitrary law, for applying the combined control principles. The experimental sample of astatic state and load observer, based on TI DSP-microcontroller, have been developed, tested and analyzed.

\section{ACKNOWLEDGEMENT}

The research was supported by Russian federal target program "Scientific, research and educational staff of innovation Russia".

\section{REFERENCES}

[1] D.J. Atkinson, P.P. Acarnley, and J.W. Finch, Observers for induction motor state and parameter estimation. IEEE Transactions on Industrial Applications IA-27, pp. 1119-1127, 1991.

[2] T. Du and M.A. Brdys, Shaft, speed, load torque and rotor flux estimation of induction motor drive using an extended Luenberger observer. IEE EMD, pp. 179-184, 1993.

[3] T. Okuyama, N. Fujimoto, T. Matsui, and Y. Kubota, A high performance speed control scheme of induction motor without speed and voltage sensors. IEEE IAS Annual Meeting, Denver, pp. 106-110, 1986.

[4] D.G. Luenberger, An introduction to observers. IEEE Transactions on Automatic Control vol.16, pp. 596-602, 1971.

[5] N. T. Kuzovkov, Modal control and observers. Moscow: Machine building, 1976.

[6] A. R. Kolganov, S.V. Burenin, Algorithms and functional design programs of control systems of electromechanical objects. Ivanovo: Ivanovo State Power University Press, 1997.

[7] P. Vas, Sensorless vector arid direct torque control. London: Oxford University Press, 1998.

[8] S. Beierke, P. Vas, B. Simor, and A.F. Stronach, DSP-controlled sensorless a.c. vector drives using the extended Kalman filter. PCIM, Nurnberg, pp. 31-42, 1997

[9] A. Vinogradov, V. Glazunov, N. Gnezdov, and S.K. Lebedev, "Analysis of design variants of regulators and observers of ACS with elastic bonds," in Universities' Izvestiya. Technology of Textile Industry, vol.5, pp. 87-93, Ivanovo, 2003.

[10] S.K. Lebedev, A.A. Korotkov, "Algorithms of synthesis for the electric drives load observers," in ISPU Vestnik, vol.3, pp. 5-8, Ivanovo, 2009.

[11] N. Gnezdov, A. Korotkov, and S.K. Lebedev, "Selecting the parameters of standard distributions in the electric drives synthesis," in ISPU Vestnik, vol.3, pp. 14-16, Ivanovo, 2008.
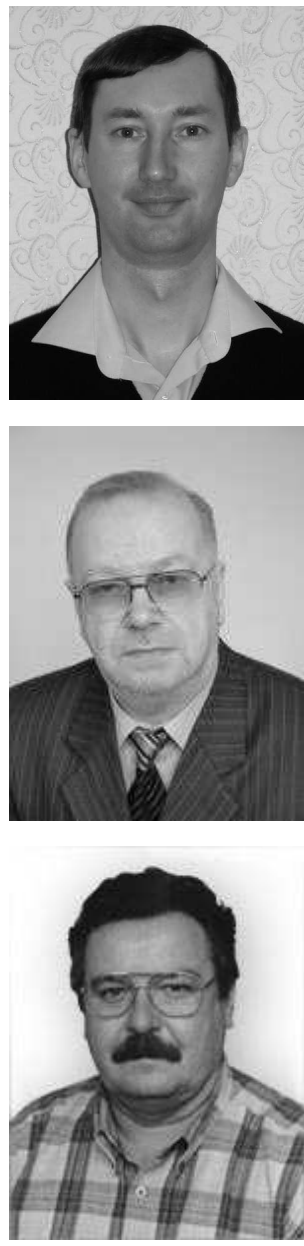

Nikolay E. Gnezdov has received Master's degree in engineering in 2002 in ISPU. In 2009 he has also received Candidate's degree in electrical engineering for the dissertation "Multilinked Induction Electric Drive with Vector Control and Non-rigid Mechanics." Associate Professor of Electric Drive (ED) Department, ISPU, Ivanovo, Russia. Scientific interests: State Control of Electromechanotronic Systems with rigid and elastic mechanics, Communication tools of modern electric drives. E-mail: gnezdov@drive.ispu.ru

Aleksey R. Kolganov has received Engineer's degree in Ivanovo Power Institute In 1974. In 1981 he has received Candidate's degree and in 2000 - also Doctor's degree for the dissertation "Technology of Automation of Functional Designing and Analysis of Complex Dynamic Objects." Head of ED Department, ISPU, Ivanovo, Russia. Scientific interests: Design and realization of computer technologies of automation of functional designing and imitating modelling of electromechanical systems. E-mail: klgn@drive.ispu.ru

Sergey K. Lebedev has received Engineer's degree in Ivanovo Power Institute in 1975. In 1986 he has received also Candidate's degree in electrical engineering for the dissertation "Design and Research of Devices of Coordinates and Parameters Definition for the Induction Electric Drive with Vector Control based on Current and Stator Voltage Sensors." Associate Professor of ED Department, ISPU, Ivanovo, Russia. Scientific interests: Control systems of the alternating current electric drives, State regulators and observers for electromechanical systems. E-mail: lebedev@drive.ispu.ru 